\title{
INFLUENCIA DEL ESTADO NUTRICIONAL EN LAS MALOCLUSIONES EN NIÑOS DE 6 A 12 AÑOS DE LAS INSTITUCIONES EDUCATIVAS DE LA MICRORED FRONTERA DEL CENTRO DE SALUD POCOLLAY- TACNA, 2012
}

\author{
INFLUENCE OF NUTRITIONAL STATUS IN CHILDREN \\ MALOCCLUSIONS 6 TO 12 YEARS OF EDUCATIONAL INSTITUTIONS OF \\ THE BORDER MICRORED POCOLLAY HEALTH CENTER-TACNA, 2012
}

\author{
Jose Luis Pacheco Torre ${ }^{1}$
}

\begin{abstract}
RESUMEN
La investigación de campo se realizó a nivel descriptivo - relacional, de corte transversal, cuantitativo, observacional, para conocer el estado nutricional y maloclusiones de niños de 6 al2 años de las Instituciones Educativas de la Microred Frontera - Centro de Salud Pocollay, Tacna- Perú. La población estudiada fue de 317 escolares de ambos sexos, que constituyen el 100\%, conformado por alumnos del primer al sexto grado de primaria y del primero de secundaria, perteneciente a las Instituciones Educativas Manuel Flores Calvo y Santa María Eufrasia del distrito de Pocollay, provincia deTacna en el año 2012.
\end{abstract}

Palabras clave: estado nutricional, IMC, maloclusiones, edad, peso y talla.

\begin{abstract}
Field research was conducted at the descriptive level - relational, cross-sectional, quantitative, observational, to meet the nutritional and malocclusions in children from 6 to 12 years of Educational Institutions of the Microgrid Frontera - Pocollay Health Center, Tacna, Peru. The population consisted of 317 students of both sexes, which constitute $100 \%$, consisting of students from first to sixth grade and seventh grade, educational institutions belonging to Manuel Flores Calvo and St. Mary Euphrasia Pocollay district, Tacna province in 2012.
\end{abstract}

Keywords: nutritional status, BMI, malocclusion, age, weight and size.

\section{INTRODUCCIÓN}

La presente investigación tiene como propósito establecer la relación entre el estado nutricional y la incidencia de maloclusiones en escolares de 6 a 12 años, pertenecientes al Distrito de Pocollay, de la Micro Red Frontera.

Una gran parte de las causas no genéticas que ocasionan las maloclusiones se producen después del nacimiento.

Las condiciones socioeconómicas influyen en la nutrición, interfiriendo en la secuencia normal y en el desarrollo del complejo craneofacial, especialmente la cara, los maxilares y los dientes.

La población estudiada fue de 317 escolares de ambos sexos.

\section{Objetivo}

Como objetivo general nos propusimos relacionar el estado nutricional con las maloclusiones que presentan los alumnos de 6 a 12 años de las Instituciones Educativas de la Microred Frontera del Centro de Salud Pocollay; como objetivos específicos, conocer el estado

Cirujano Dentista. Facultad de Ciencias de la Salud de la UNJBG. 
nutricional y determinar las maloclusiones que presentan los niños de las instituciones educativas estudiadas.

\section{MATERIAL Y MÉTODOS}

\subsection{Ubicación}

El trabajo se realizó en las Instituciones Educativas Manuel Flores Calvo, Santa María Eufrasia del distrito de Pocollay, en el año 2012.

\subsection{Metodología}

Se trata de una investigación de campo a nivel descriptivo - relacional, de corte transversal, cuantitativo, observacional y de cohortes, en relación al estado nutricional y maloclusiones en niños de 6 a 12 años de las Instituciones Educativas de la Microred Frontera CSP de Tacna en el año 2012.

La población tanto de primaria como de secundaria, la conforman 317 escolares de ambos sexos y constituyen el $100 \%$. La edad oscila entre 6 a 12 años. Se aplicó las tablas de valoración nutricional antropométricas para varones y mujeres de 5-19 años, establecidas por el Centro Nacional de Alimentación y Nutrición, auspiciadas por el Ministerio de Salud (MINSA) e Intituto Nacional de Salud (INS), y la ficha epidemiológica de maloclusiones dada por la Universidad Católica de Santa María (UCSM) de Arequipa.

\section{RESULTADOS}

Tabla $\mathrm{N}^{\circ}$ 01: Distribución numérica y porcentual de los alumnos según sexo.

\begin{tabular}{|c|c|c|}
\hline Sexo & Frecuencia & Porcentaje \\
\hline Femenino & 230 & $72.56 \%$ \\
\hline Masculino & 87 & $27.44 \%$ \\
\hline Total & 317 & $100.00 \%$ \\
\hline
\end{tabular}

Fuente: propia

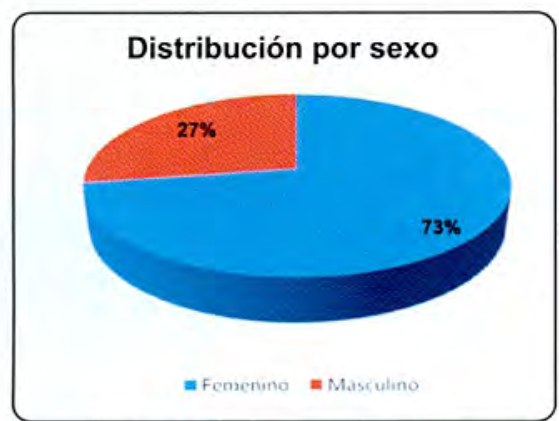

Gráfico $\mathrm{N}^{\circ} 01$ :Frecuencia de distribución por sexo.
De los 317 alumnos evaluados, 230 corresponde al sexo femenino $(72.56 \%)$, mientras que 87 , al sexo masculino $(27.44 \%)$.

Tabla $\mathrm{N}^{\circ}$ 02: Distribución numérica y porcentual de los alumnos según talla.

\begin{tabular}{|c|c|c|}
\hline Talla/edad & Frecuencia & Porcentaje \\
\hline Talla Baja & 30 & $9.46 \%$ \\
\hline Normal & 268 & $84.54 \%$ \\
\hline Talla Alta & 19 & $5.99 \%$ \\
\hline Total & 317 & $100.00 \%$ \\
\hline
\end{tabular}

Fuente: Propia

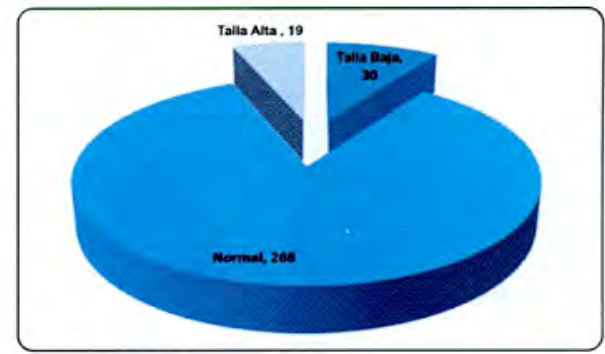

Gráfico $\mathrm{N}^{\circ}$ 02: Frecuencia por talla

Del total de alumnos evaluados, 268 corresponden a una talla normal (84.54 \%), mientras que 30 , a una talla baja $(9.46 \%)$ y el 5.99 $\%$ corresponde a una talla alta que engloba solo a 19 niños.

Tabla $\mathbf{N}^{\circ}$ 03: Distribución numérica y porcentual de los alumnos según índice de masa corporal.

\begin{tabular}{|c|c|c|}
\hline IMC & Frecuencia & Porcentaje \\
\hline Delgadez & 7 & $2.21 \%$ \\
\hline Normal & 238 & $75.08 \%$ \\
\hline Obesidad & 72 & $22.71 \%$ \\
\hline Total & 317 & $100.00 \%$ \\
\hline
\end{tabular}

Fuente: propia

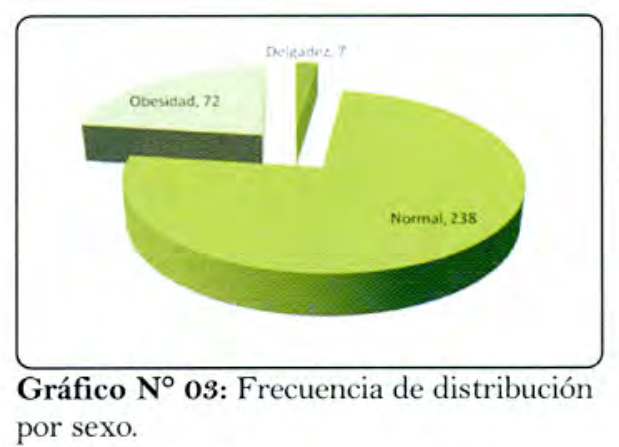

De los 317 alumnos evaluados, 7 casos presentan delgadez $(2.21 \%), 72$ tienen obesidad $(22.71 \%)$ y 238 casos corresponde a una IMC normal $(75.08 \%)$. 
Tabla $\mathrm{N}^{\circ}$ 04: Distribución numérica y porcentual de maloclusiones.

\begin{tabular}{|l|c|c|}
\hline Maloclusion & Frecuencia & Porcentaje \\
\hline Clase I & 268 & $84.54 \%$ \\
\hline Clase II-1 & 25 & $7.89 \%$ \\
\hline Clase II-2 & 8 & $2.52 \%$ \\
\hline Clase III & 16 & $5.05 \%$ \\
\hline Total & 317 & $100.00 \%$ \\
\hline
\end{tabular}

Fuente: Propia

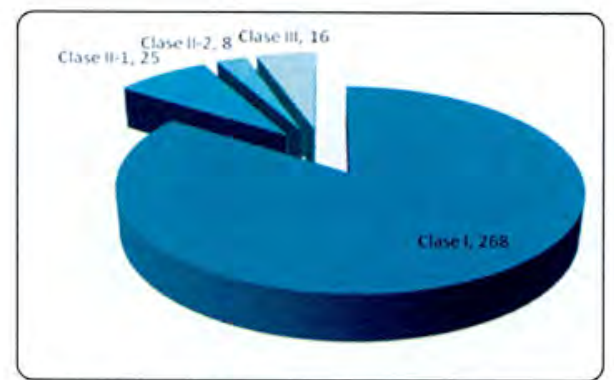

Gráfico $\mathrm{N}^{\circ}$ 04: Diagnóstico del maloclusiones.

De la población evaluada, 268 presentan maloclusión de Clase I (84.54\%); 33 alumnos, maloclusión Clase II (10.41 \%) y 16 alumnos, maloclusión Clase III ( $5.05 \%)$.

Tabla $\mathrm{N}^{\circ}$ 05: Distribución por edad, peso y talla, según media y desviación estándar.

\begin{tabular}{|l|c|c|c|c|}
\hline \multirow{2}{*}{ Variables } & \multicolumn{2}{|c|}{ Femenino } & \multicolumn{2}{c|}{ Masculino } \\
\cline { 2 - 5 } & \multicolumn{2}{|c|}{$\mathrm{n}=290$} & \multicolumn{2}{c|}{$\mathrm{n}=87$} \\
\cline { 2 - 5 } & Media & D.E & Media & D.E \\
\hline Edad(Años) & 9.44 & 2.03 & 9.49 & 2.04 \\
\hline Peso(kg) & 33.96 & 12.08 & 34.28 & 9.90 \\
\hline Talla(mt) & 1.34 & 0.14 & 1.33 & 0.11 \\
\hline
\end{tabular}

Fuente: Propia

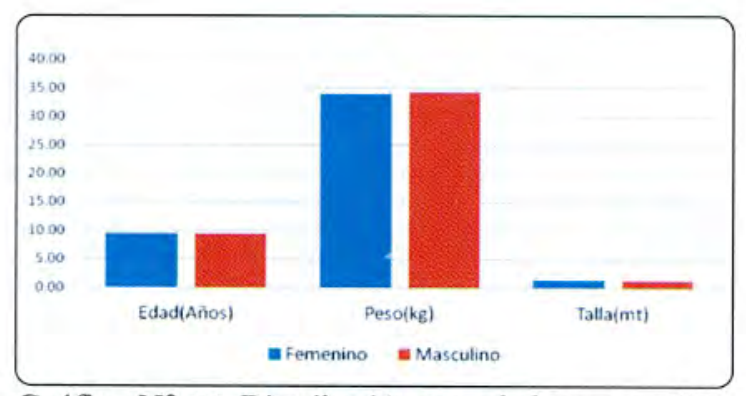

Gráfico $\mathrm{N}^{\circ}$ 05: Distribución por edad, peso y talla.

La media para la edad de sexo femenino es 9.44; para los de edad de sexo masculino, 9.49. La media para el peso femenino es 33.96; para el masculino, 34.28. La media para la talla de sexo femenino es 1.34; para el masculino, 1.33 .
Tabla Nº6: Relación de maloclusiones según talla.

\begin{tabular}{|c|c|c|c|c|c|}
\hline & \multicolumn{4}{|c|}{ MALOCLUSIONES } & TOTAL \\
\hline & Clase I & Clase II-1 & Clase II-2 & Clase III & CANT \\
\hline Talla Baja & 21 & 20 & 7 & 12 & 60 \\
\hline Normal & 229 & 1 & 0 & 0 & 290 \\
\hline Talla Alta & 18 & 4 & 1 & 4 & 27 \\
\hline Total & 268 & 25 & 8 & 16 & 317 \\
\hline
\end{tabular}

Fuente: Propia

El valor de chi cuadrado en el siguiente gráfico es de $\mathrm{p}=0.22$.

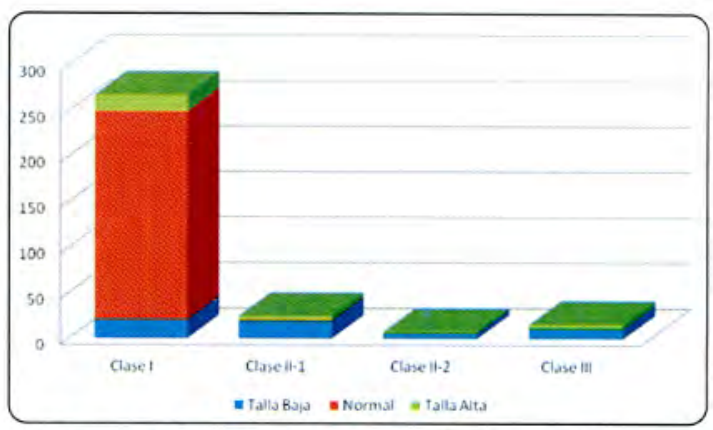

Gráfico $\mathrm{N}^{\circ}$ 06: Relación maloclusión - talla

No hay relación entre la talla y las maloclusiones. La mayoría presenta talla normal y maloclusión Clase I.

Tabla $\mathbf{N}^{\circ}$ 07: Relación de maloclusiones según IMC.

\begin{tabular}{|c|c|c|c|c|c|}
\hline & \multicolumn{4}{|c|}{ MALOCLUSIONES } & \multirow{2}{*}{$\begin{array}{l}\text { TOTAI } \\
\text { CANT }\end{array}$} \\
\hline & Clase I & Clase II-1 & Clase II-2 & Clase III & \\
\hline Delgadez & 4 & 2 & 0 & 1 & 7 \\
\hline Normal & 209 & 14 & 5 & 10 & 298 \\
\hline Obesidad & 55 & 9 & 3 & 5 & 72 \\
\hline TOTAL & 268 & 25 & 8 & 16 & $\$ 17$ \\
\hline
\end{tabular}

Fuente: Propia

El valor de chi cuadrado en el siguiente gráfico es de $\mathrm{p}=0.72$.

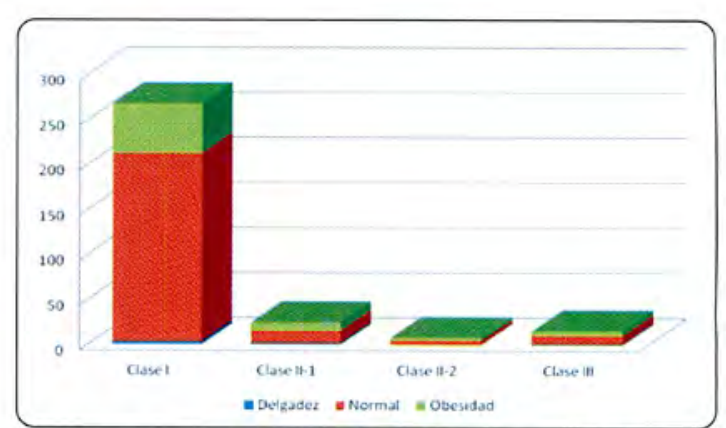

Gráfico $\mathrm{N}^{\circ}$ 07: Relación de maloclusión - IMC.

No hay relación entre IMC y maloclusiones, observándose que la mayoría presenta peso normal con una maloclusión de Clase I, y un pequeño porcentaje de obesidad con maloclusión de Clase I. 
Tabla $N^{\circ}$ 08: Relación de maloclusión según sexo.

\begin{tabular}{|c|c|c|c|c|c|}
\hline & \multicolumn{4}{|c|}{ MALOCLUSIONES } & \multirow{2}{*}{$\begin{array}{c}\text { TOTAL } \\
\text { CANT }\end{array}$} \\
\hline & Clase I & Clase II-1 & Clase II-2 & Clase III & \\
\hline Femenino & 195 & 20 & 5 & 10 & 230 \\
\hline Masculino & 79 & 5 & 3 & 6 & 87 \\
\hline Total & 268 & 25 & 8 & 16 & $\$ 17$ \\
\hline
\end{tabular}

Fuente: Propia

El valor de chi cuadrado en el siguiente gráfico es de $\mathrm{p}=0.589$.

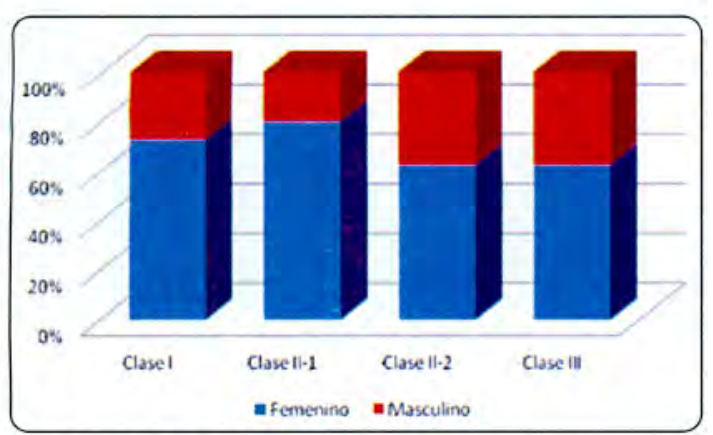

Gráfico $\mathrm{N}^{\circ}$ 08: Relación maloclusión - sexo.

No hay relación entre sexo y maloclusiones.

\section{IV.DISCUSIÓN}

Es política del Estado combatir la desnutrición crónica, en el binomio madre - niño, estableciendo la seguridad alimentaria con la creación del Ministerio de Desarrollo e Inclusión Social, donde se van a articular todos los Programas Sociales, que aseguren que los niños incorporen los alimentos necesarios para mantener su salud y elevar su calidad de vida, a través de los comedores populares, cunamás, etc.

En esta investigación, el porcentaje de niños con delgadez y talla baja es mínimo, ya que la Región Tacna no se encuentra en el bolsón de la extrema pobreza, pero pueden presentar posteriormente problemas de crecimiento y desarrollo.

Un mayor número de niños se encuentran con obesidad, de no controlarse, ocasionará trastornos en el metabolismo celular y aumentará el riesgo de padecer trastornos cardiovasculares (dislipidemias) y metabólicos, como diabetes, hipertensión, apnea del sueño, complicaciones ortopédicas y consecuencias de orden psíquico.

\section{CONCLUSIONES}

- El estado nutricional de los alumnos de 6 a 12 años de las I.E. de la Micro Red Frontera del C.S. Pocollay fue analizado en base a los indicadores de IMC y talla para la edad, según las tablas de la OMS, de donde se pudo concluir que el mayor porcentaje se encuentra con un IMC-NORMAL.

- Las maloclusiones que presentaron los niños de 6 a 12 años en mayor porcentaje es de Clase I.

- Para este trabajo de investigación se aplicó el chi cuadrado, relacionando el estado nutricional con las maloclusiones, indicándonos que en estos casos no presentan relación alguna, ya que la mayoría de niños se encuentran con IMC-NORMAL y con una maloclusion de Clase I.

\section{RECOMENDACIONES}

Los estudios posteriores deberían realizarse en niños de extrema pobreza, donde su IMC sea de delgadez, talla baja; ya que según estudios internacionales, ellos se encuentran predispuestos a sufrir de déficit en el desarrollo cráneo facial.

Es imprescindible que el MINSA realice un seguimiento del estado nutricional del niño, pues este influye grandemente en la salud bucal del mismo.

Es necesario que la estrategia sanitaria de salud bucal del MINSA incida en el tema de nutrición del binomio madre-niño, ya que con dicha incidencia se mejorará el estilo de vida de nuestra población en extrema pobreza.

\section{REFERENCIAS BIBLIOGRÁFICAS}

Cardenas J.M y col. "La profesión Odontológica frente al estado actual y futuro de la salud oral en la población pediátrica y adolescente en Colombia. Revista CES Odontológica. Vol.9 $\mathrm{N}^{\circ} 2-1996$.

Rivera 1. y col. "Estudio de caries y maloclusiones en niños de un sector de salud de la ciudad de Santa Clara". Rev. Cub. de Estom. Vol. $20 \mathrm{~N}^{\circ} 21983$.

Giunta J.L. (1997). Patología Bucal. Sra Edición. Editorial Interamericana. México D.F.

López Suárez. (2005). Fundamentos de Nutrición Normal. Edit. Atenea. Buenos Aires.

Bueno, M; Sarria, A; Perez, J. Nutrición en pediatría. Edit ERGON. Madrid 2003. 11-6.

Majan, L. K., \& Escott-Stump, S. (1996). Nutrición y Dietoterapia de Krause (9na. ed., 
pp. 321-370). México: McGraw-Hill Interamericana.

Bistrian Br, Blackburn GL, et al.: Prevalence of malnutrition in general medical patients. JAMA 1976; 235: 1567-70.

Chandra RK. Nutrition, inmunity and infection: present knowledge and future directions. Lancet 1983; 1:688-91.

Herrmann FR, Safran C, et al.: Serum albumin level on admission as a predictor of death, length of stay and readmission. Arch Intern Med 1992; 152: 125-30.

Roldán JP, Pérez I, et al.: Malnutrición en pacientes hospitalizados: estudio prospectivo y aleatorio. Nutr Hosp 1995, 10: 192-198

Canut Brusola, José A. (1989). Ortodoncia Clínica. Salvat. Barcelona.

Graber, Thomas y Vanarsdall, Robert. (1997). Ortodoncia Principios Generales y Técnicas. Segunda Edición. Editorial Médica Panamericana. Buenos Aires.
Moyers, Robert E. (1992). Manual de Ortodoncia. Cuarta Edición. Editorial Médica Panamericana. Buenos Aires.

Clemens Man Hattsberber (1996). MD DDS $\mathrm{Ph}$. and Seldenbwich wolter PH.D. Insbruck Austria American Journal, Orthodontic and Dentofacial Orthopedics January, 1996

Larry Rosado Linares (1997) Diseño E Implementacion Del Protocolo Investigativo En Odontoestomatologia.

Jesús Calatayud y Gonzalo Martín, Bioestadística en la Investigación Odontológica.

\section{Correspondencia:}

Luis Pacheco Torre

Ciudad Universitaria fundo "Los Granados" Av. Miraflores s/n Tacna - Perú

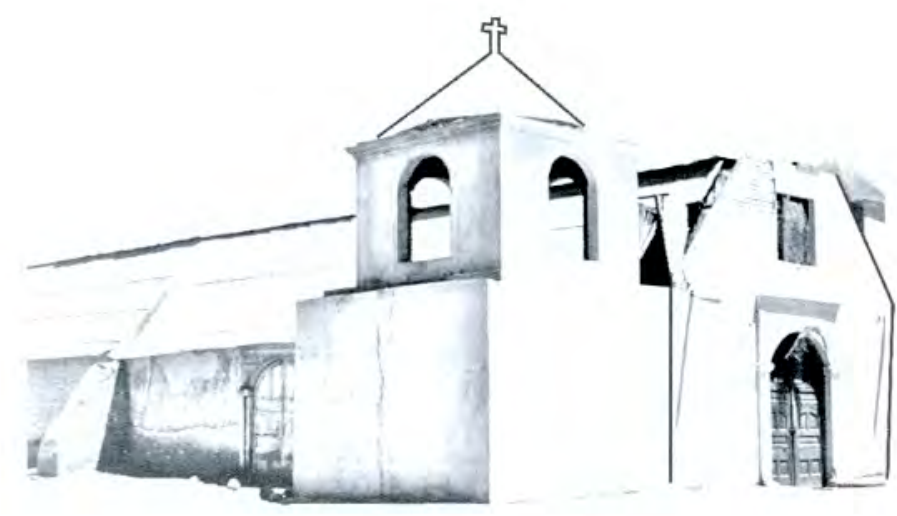

Iglesia de Buena Vista 